\title{
Tumor Targeted Curcumin Delivery by Folate-Modified MPEG-PCL Self-Assembly Micelles for Colorectal Cancer Therapy
}

This article was published in the following Dove Press journal: International Journal of Nanomedicine

\author{
Yuzhu Hu${ }^{1-3}$ \\ Yihong $\mathrm{He}^{1-3}$ \\ Jianrui Ji' \\ Songping Zheng ${ }^{\mathrm{I}-3}$ \\ Yongzhong Cheng (1D ${ }^{1-3}$ \\ 'Department of Neurosurgery and \\ Institute of Neurosurgery, State Key \\ Laboratory of Biotherapy, West China \\ Hospital, West China Medical School, \\ Sichuan University and Collaborative \\ Innovation Center for Biotherapy, \\ Chengdu 61004I, People's Republic of \\ China; ${ }^{2}$ Department of Medical \\ Oncology, Cancer Center, West China \\ Hospital, West China Medical School, \\ Sichuan University, Chengdu 61004I, \\ People's Republic of China; ${ }^{3}$ Department \\ of Neurosurgery, West China Hospital, \\ West China Medical School, Sichuan \\ University, Chengdu 61004I, People's \\ Republic of China
}

Correspondence: Songping Zheng; Yongzhong Cheng

Department of Neurosurgery, West China Hospital, West China Medical School, Sichuan University, Chengdu 61004I, People's Republic of China Tel +862885164063

Email 136I808805I@I63.com; chengyz@scu.edu.cn
Introduction: Curcumin (Cur) is a natural extract of Asian spice Curcumin longa, showing multi-targeting capability and low toxicity in anti-tumor activities. The low bioavailability restricts its application as a therapeutic agent. Folate (FA) receptors are highly expressed in many malignant tumors while low expressed in normal tissue. Herein, we developed a selfassembled FA modified MPEG-PCL micelle to incorporate Cur (FA/Nano-Cur) and applied it for colorectal cancer therapy.

Methods: We prepared FA/Nano-Cur micelles and identified their characteristics. The drug release behavior, pharmacokinetics and in vitro anti-tumor activities of FA/Nano-Cur were studied. Furthermore, the in vivo anti-tumor ability assessment and anti-tumor mechanisms investigation were carried out in murine colorectal cancer model.

Results: FA/Nano-Cur micelles had an average particle size of $30.47 \mathrm{~nm}$. Elongated $T_{1 / 2}$ and larger AUC were found in FA/Nano-Cur group than that in the Free Cur group. MTT assay and apoptotic study indicated the growth inhibitory effect and pro-apoptotic effect of FA/ Nano-Cur were the most significant among all treatments. Moreover, the in vivo study demonstrated that FA/Nano-Cur micelles exhibited a much stronger effect to suppress tumor growth, promote tumor apoptosis and attenuate tumor angiogenesis than Free Cur and Nano-Cur micelles.

Conclusion: The present study demonstrated FA/Nano-Cur micelles might be a promising therapeutic agent in colorectal cancer treatment with distinctive advantages of improved bioavailability, sustained drug release, tumor-targeted delivery and low toxicity.

Keywords: curcumin, colorectal cancer, folate, nanoformulation, apoptosis, angiogenesis

\section{Introduction}

Colorectal cancer (CRC) is the third most common tumor and one of the main causes of cancer-related death among both men and women worldwide. ${ }^{1}$ The highest incidence of CRC is reported in Alaska natives and blacks, while the lowest is in Asian Pacific Islanders, which is closely related to age and so-called western lifestyle. $^{2-4}$ In recent years, the morbidity and mortality rates of CRC have begun to decrease as a result of lifestyle changes in pathogenetic reasons (e.g., decreased smoking and alcohol consumption, body shaping and reduced red meat intake), the application of sigmoidoscopy and colonoscopy, as well as improved treatment. ${ }^{4-9}$ Unfortunately, the patients who developed distant metastasis had a five-year survival of only about $11.7 \% .^{10}$ The treatment options for CRC included surgery, chemotherapy, targeted therapy, radiotherapy and immunotherapy. Chemotherapy 
as the principal treatment approach has some adverse effects involving gastrointestinal tract toxicity, bone marrow suppression, neurotoxicity, liver and kidney damage and other tissue damages. ${ }^{11}$ Moreover, current treatment exhibited high recurrence and poor prognosis. Therefore, it is increasingly important to explore more efficient and less toxic treatment approach for CRC.

Phytotherapy has received more and more attention in the field of medicine, focusing mainly on herbal active pharmaceutical ingredients. ${ }^{12}$ Curcumin (Cur), an active polyphenolic compound extracted from turmeric (Curcuma longa L.), was found to possess beneficial properties including anti-oxidant, anti-inflammatory, antihyperlipidemic, anti-diabetic, anti-microbial and powerful anti-tumor effects. ${ }^{13-18}$ Among these, the anti-tumor effect of Cur is the most popular at present. It is reported that Cur had inspiring anti-tumor abilities in melanoma, gastrointestinal, genitourinary, pancreatic, breast, lung, haematological, head and neck, neurological carcinomas and sarcoma. ${ }^{15,19-23}$ What's more, Cur proved to be nontoxic and safe in animal studies, making it a promising drug agent. ${ }^{24}$ Despite these advantages, Cur has not yet been approved as a therapeutic agent for cancer. The main drawback is poor bioavailability. ${ }^{25-27}$ Some pharmacokinetic researches have indicated that the poor bioavailability of unformulated Cur is mainly attributed to low water solubility, fast metabolism and rapid systemic clearance, which limit its therapeutic efficiency. ${ }^{27}$ Thereupon, we aim to develop a new dosage form of Cur to overcome the poor bioavailability and improve therapeutic efficacy in cancer treatment.

Nanotechnology is playing a significant role in drug delivery systems nowadays. Nanoparticles, such as liposomes, polymeric micelles, can encapsulate water-insoluble drugs as vectors and protect them from enzymatic and hydrolytic degradation. ${ }^{28-30}$ Methoxypoly (ethylene glycol)-poly ( $\varepsilon$-caprolactone) (MPEG-PCL) are biodegradable, amphiphilic diblock copolymers. ${ }^{31}$ When the copolymers are in aqueous solution, the hydrophobic PCL segments can spontaneously assemble to form a core enclosing water-insoluble drug; meanwhile, the hydrophilic PEG segments are encircled by water to form a shell-like structure. ${ }^{32}$ The hydrophobic drugs loaded MPEG-PCL copolymers are capable of sustaining drug release and improving drug bioavailability. ${ }^{33,34}$ Moreover, the amphiphilic MPEG-PCL micelles have biodegradability and nanoscale properties, making them ideal drug carriers for systemic administration.
Usually, the activity of pharmaceutical agent is nonselective, and the agent can be inactivated or induce adverse effects on normal tissues and organs. ${ }^{35}$ Folate (FA) is a member of water-soluble vitamin B family, which has low immunogenicity. FA receptors are glycoproteins fixed to the cell membrane by glycosyl phosphatidyl inositol (GPI), showing high affinity to FA and FA modified drug formulation, so the drug can be transferred into cells through endocytosis. $^{36}$ FA exhibits distinguished advantage in increasing the specificity of anti-cancer drug vehicle because the FA receptors are highly expressed in various malignant tumors including colon, brain, lung and breast carcinomas, but low expressed in normal tissues. ${ }^{37-40}$ Therefore, FA conjugated nanoformulation might be useful to realize active tumor-targeted drug delivery, sustain drug release, as well as reduce adverse effect. In this research, we prepared the FA/ Nano-Cur formulation and compared its therapeutic effect with Free Cur and Nano-Cur formulation in the treatment of CRC. Our in vitro and in vivo results demonstrated that the Cur-loaded FA/MPEG-PCL micelles might be a promising therapeutic approach for CRC.

\section{Materials and Methods Materials and Reagent}

Curcumin, FA, MPEG (molecular weight=2000D), $\varepsilon$ caprolactone $(\varepsilon-C L)$ (molecular weight=2000D), 3-(4, 5-dimethylthiazol-2-yl)-2, 5-diphenyl tetrazolium bromide (MTT) were purchased from Sigma, USA. Roswell Park Memorial Institute (RPMI) 1640 and fetal bovine serum (FBS) were purchased from Gibco, USA. Methanol and acetic acid (HPLC grade) were purchased from Fisher Scientific, UK. 4', 6-diamidino-2-phenylindole (DAPI) and hematoxylin and eosin (HE) dye from Beyotime Biotechnology, China. Dimethyl sulfoxide (DMSO) and acetone were purchased from KeLong Chemicals, China. Antibodies used include: rabbit anti-mouse cluster of differentiation 31 (CD31) polyclonal antibody, rabbit antimouse proliferating cell nuclear antigen (PCNA) antibody, and horseradish peroxidase (HRP)-conjugated secondary antibody (Servicebio, China).

Murine CT26 colon carcinoma cells were purchased from American Type Culture Collection (ATCC, USA) and cultured in RPMI 1640 culture medium containing $10 \%$ FBS, $100 \mathrm{U} / \mathrm{mL}$ penicillin, and $100 \mu \mathrm{g} / \mathrm{mL}$ streptomycin. Cells were maintained in a humidified chamber at $37^{\circ} \mathrm{C}$ in an atmosphere containing $5 \% \mathrm{CO}_{2} .6-8$ weeks female $\mathrm{BALB} / \mathrm{C}$ mice were obtained from the Animal 
Center Laboratory of Beijing HFK Bioscience (China) and provided with sterilized water and chow. All animal experimental procedures were approved by the Institutional Animal Care and Treatment Committee of Sichuan University and conducted according to the Institutional Animal Care and Use Guidelines.

\section{Preparation and Characterization of FA/ Nano-Cur Micelles}

According to the previous reports, ${ }^{33}$ MPEG-PCL diblock copolymer was synthesized by ring-opening polymerization of MPEG and $\varepsilon-C L$ catalyzed by $\mathrm{Sn}(\mathrm{Oct})_{2}$. Briefly, an equal amount of MPEG, $\varepsilon$-CL were introduced into a glass ampoule with the addition of $\mathrm{Sn}(\mathrm{Oct})_{2}(0.5 \% \mathrm{w} / \mathrm{w})$ under nitrogen protection. The mixture reactant was maintained at $130^{\circ} \mathrm{C}$ for $6 \mathrm{~h}$. The crude product was then dissolved in dichloromethane and purified by precipitation in ice-cooled diethyl ether followed by vacuum drying for further use. FA $(100 \mathrm{mg})$ and $\mathrm{NH}_{2}$-PEG-PCL (200 mg) dissolved in $4 \mathrm{~mL}$ of dimethylsulfoxide (DMSO) were introduced into a flask, with the addition of DMAP $(150 \mathrm{mg})$ and DCC $(130 \mathrm{mg})$. After agitation at $37^{\circ} \mathrm{C}$ under nitrogen for $10 \mathrm{hrs}$, the synthesized copolymer solution was dialyzed and dried under vacuum. All obtained products were stored in a desiccator.

Cur $(10 \mathrm{mg})$, MPEG-PCL (80 mg) and FA/MPEG-PCL $(10 \mathrm{mg})$ were dissolved in $3 \mathrm{~mL}$ acetone in a round bottom flask. Next, the acetone was removed by vacuum evaporation under $55^{\circ} \mathrm{C}$ water bath and a yellow film was formed on the bottom of the flask. PBS was added to hydrate the film and slowly stirred. The un-encapsulated drug and polymerized copolymer were filtered and removed with a $0.22-\mu \mathrm{m}$ filter. Blank micelles were prepared following the same procedure. The particle size and zeta potential of FA/Nano-Cur micelles were determined by dynamic light scattering (Malvern NanoZS 90, UK). The morphology of FA/Nano-Cur was observed under a transmission electron microscope (TEM) after phosphotungstic acid staining (Hitachi H-6009IV, Japan). The content of Cur in FA/Nano-Cur was determined by highperformance liquid chromatography (HPLC). The encapsulation efficiency (EE) and drug loading (DL) of FA/Nano-Cur micelles were calculated according to the equations: drug loading (DL) $=$ experimental Cur loading/total micelle mass $\times 100 \%$ (a), encapsulation efficiency $(\mathrm{EE})=$ experimental Cur loading/theoretical Cur loading $\times 100 \%$ (b).

The in vitro drug release behavior of Cur from Free Cur, Nano-Cur and FA/Nano-Cur were conducted using the dialysis method. $0.5 \mathrm{~mL}$ of Free Cur in DMSO solution, Nano-
Cur solution and FA/Nano-Cur solution was placed in the dialysis bag (molecular weight cut-off, $3.5 \mathrm{kDa}$ ), respectively. Dialysis bags were incubated in $30 \mathrm{~mL}$ of phosphatebuffered solution $(\mathrm{pH}=7.4)$ containing Tween-80 $(0.5 \mathrm{wt} \%)$ at $37^{\circ} \mathrm{C}$ with mild shaking. The incubation medium was replaced with fresh incubation medium at different time points, respectively. The drug release profiles of Cur from different formulations were studied by HPLC assay.

\section{Pharmacokinetic Study}

Firstly, a catheter was introduced into the jugular vein of rats $24 \mathrm{hrs}$ before drug administration. The rats were then assigned into three groups and received different drugs: Free Cur group (50 mg/kg Cur), Nano-Cur group (50 mg/ $\mathrm{kg}$ Cur) and FA/Nano-Cur ( $50 \mathrm{mg} / \mathrm{kg}$ Cur) group. At the indicated interval, the blood was collected from the catheter in the jugular vein. The plasma of each blood sample was extracted and lyophilized. The dried plasma was dissolved in methanol and analyzed by HPLC.

\section{Cell Cytotoxicity Assay}

CT26 cells at the logarithmic phase were seeded in 96-well culture plates at a density of $3 \times 10^{3}$ or $5 \times 10^{3}$ cells/well and incubated for $24 \mathrm{hrs}$ at $37^{\circ} \mathrm{C}$ for cell attachment. Cells were treated with different concentrations of Free Cur, Nano-Cur and FA/Nano-Cur for $24 \mathrm{hrs}$ or $48 \mathrm{hrs}$, and then incubated with the addition of $20 \mu \mathrm{L}$ MTT solution $(5 \mathrm{mg} / \mathrm{mL})$ for $4 \mathrm{hrs}$. Next, the culture medium was aspirated carefully and $150 \mu \mathrm{L}$ DMSO was added. After gently shaking for 10 mins to dissolve the formazan, the absorbance of each well at $570 \mathrm{~nm}$ (OD570) was measured by a microplate reader (OPTImax, Molecular Dynamics, USA).

\section{Cell Necrosis and Apoptosis Assay}

Cell necrosis and apoptosis were analyzed using a commercial Propidium (PI)/fluorescein isothiocyanate (FITC) conjugated Annexin-V apoptosis detection kit (BD Pharmingen, USA). CT26 cells $\left(2 \times 10^{5}\right.$ cells/well $)$ were seeded in six-well plates for cell attachment and then incubated in culture medium containing different concentrations of Free Cur, Nano-Cur or FA/Nano-Cur. After incubated for $48 \mathrm{hrs}$, cells were collected and washed with PBS. Next, cells were re-suspended with $100 \mu \mathrm{L}$ binding buffer containing $3 \mu \mathrm{L}$ PI and $3 \mu \mathrm{L}$ Annexin-V for 15 mins. The percentages of cell necrosis and apoptosis were determined by a flow cytometer (Novocyte, USA). 


\section{Mice Model and Treatment}

In this work, the mice model of heterotopic CRC was established to evaluate the anti-tumor ability of different treatments by inoculating CT26 cells $\left(1 \times 10^{6}\right)$ in the right flank of mice. When the tumor volume reached about $100 \mathrm{~mm}^{3}$, tumor-bearing mice were randomly allocated into five treatment groups (Control, FA/Nano, Free Cur, Nano-Cur and FA/Nano-Cur) and received the treatment by intravenous injections every 2 days. Control group: normal saline (NS), FA/Nano group: equal amount of FA/Nano as FA/Nano-Cur, Free Cur group: $50 \mathrm{mg} / \mathrm{kg}$ Cur; Nano-Cur group: $50 \mathrm{mg} / \mathrm{kg}$ Cur; FA/Nano-Cur: $50 \mathrm{mg} / \mathrm{kg}$ Cur. The tumor volume and the body weight of mice were measured on indicated days during the experimental period. The calculation equation of tumor volume: $\mathrm{V}=\pi \times \mathrm{A} \times \mathrm{B}^{2} / 6$ (A: longest diameter, $\mathrm{B}$ : shortest diameter). All experimental mice were sacrificed on the 18 th days after inoculation and the tumor, peripheral blood and vital organs of mice were collected for further analysis.

\section{Immunohistochemical Analysis}

Tumor sections $(4 \mu \mathrm{m})$ were prepared and stained with PCNA and CD31 antibodies for the purpose of evaluating tumor proliferation and tumor angiogenesis in different treatment groups. Briefly, the paraffin-embedded tumor tissue sections underwent de-paraffinage and rehydration. The antigen retrieval process was conducted, followed by endogenous peroxidase inactivation with a $3 \%$ hydrogen peroxide solution. Then, the tumor sections were punched with Triton X-100 (for PCNA staining), blocked with goat serum, and sequentially incubated with PCNA antibody or CD31 antibody (Servicebio, China) and corresponding HRP conjugated secondary antibody (Servicebio, China). Finally, the labeled sections were colorated by DAB and counterstained with hematoxylin. All stained sections were observed under a light microscope at $200 \times$ magnification. Cells with positive expression of PCNA or CD31 were stained in yellow brown. The PCNA index was calculated as the amount of PCNA staining positive cells divided by the amount of total cells in five randomly selected fields. The microvascular density was calculated as the amount of CD31 staining positive cells in five randomly selected fields.

\section{Immunofluorescent Staining}

Paraffin-embedded tumor sections ( $4 \mu \mathrm{m})$ were deparaffinized and rehydrated as above. The terminal deoxynucleotidyl transferase-mediated dUTP nick-end labeling (TUNEL) staining was carried out according to the manufacturer's description of a commercially available TUNEL staining kit from Promega, USA. DNA fragmentation of apoptotic cells in tumor tissue sections from different treatment group would be labelled with FITC green fluorescence. Finally, all stained sections were examined under a fluorescence microscope at $200 \times$ magnification. The apoptotic cell percentage was calculated as the amount of TUNEL staining positive cells divided by the amount of total cells in five randomly selected fields.

\section{Safety Assessment}

The important organs including heart, liver, spleen, lung and kidney of the mice were harvested and prepared as paraffin-embedded sections $(4 \mu \mathrm{m})$. These tissue sections were stained with H\&E dye and examined under a light microscope by two independent pathologists for morphology identification. What is more, the serums of peripheral blood samples were obtained for biochemical analysis to further evaluate the toxicity of different treatments in vivo.

\section{Statistical Analyses}

Statistical analysis was performed using GraphPad Prism 6.0 software (GraphPad, USA). Data were expressed as mean \pm standard error (SD). Student's $t$-test was utilized to compare two experimental groups. $\mathrm{P}<0.05$ was considered to be statistically significant.

\section{Results}

\section{Characterization and Drug Release Behavior of FA/Nano-Cur}

A graphic scheme was shown to introduce the preparation process of FA/Nano-Cur micelles in the study (Figure 1). The amphiphilic FA/MPEG-PCL copolymers and MPEG-PCL copolymers could spontaneously gather to form a spherical like structure in aqueous solution. As is shown, waterinsoluble Cur was entrapped in the hydrophobic core of the self-assembled FA/MPEG-PCL micelle to form FA/NanoCur micelles. FA/Nano-Cur was suggested to have the capability to target the FA receptors on tumor cells, thus to enhance tumor-targeted drug delivery. The characteristics of the FA/Nano-Cur micelles were identified by the dynamic light scattering (DLS) method. The particle size of FA/NanoCur micelles was found to be $30.47 \pm 0.65 \mathrm{~nm}$ with PDI being 0.17 , indicating a very narrow particle size distribution (Figure 2A). The zeta potential is $-3.55 \mathrm{mV}$, which was slightly negative (Figure 2B). According to the observation under TEM, FA/Nano-Cur micelles were well dispersed with 


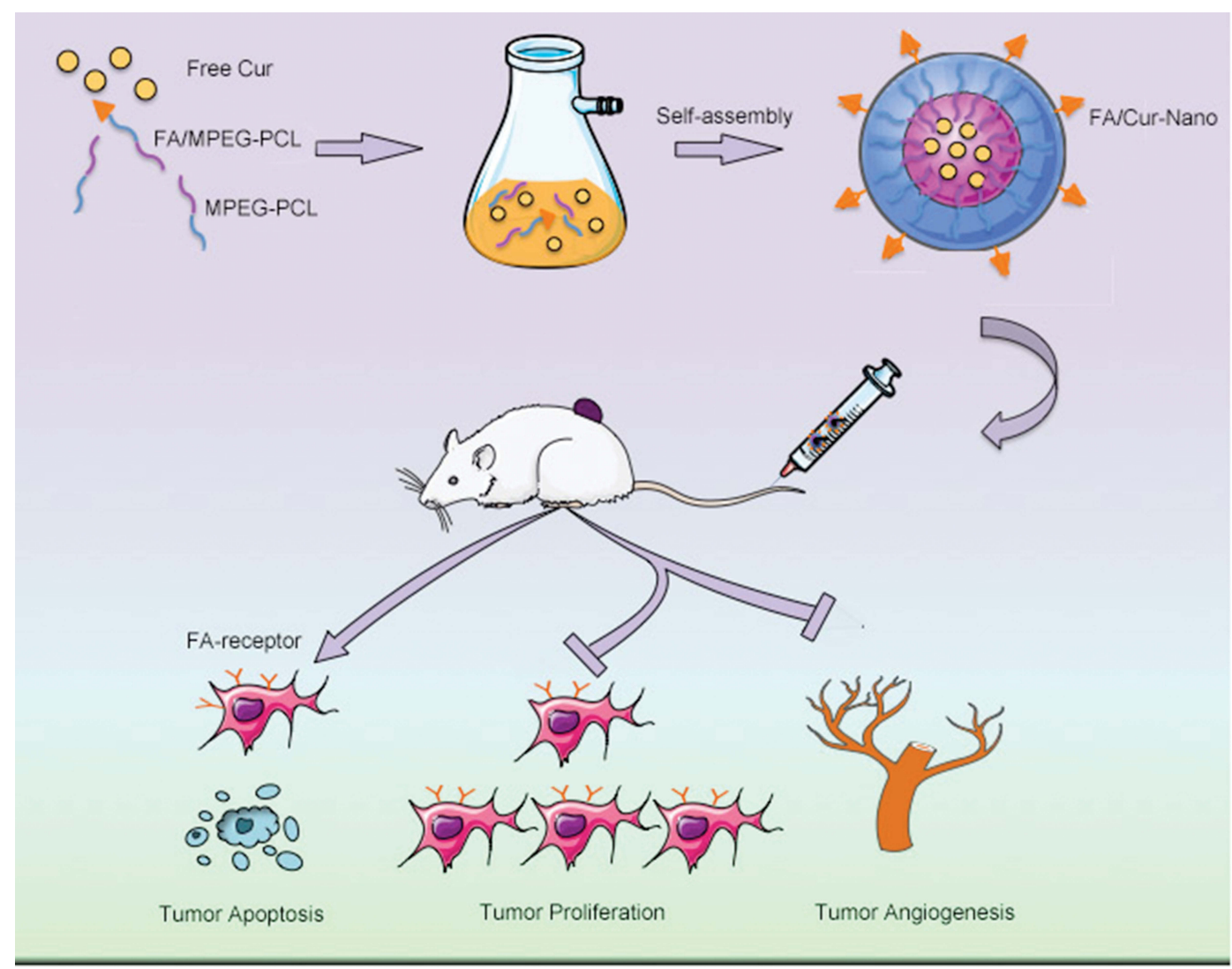

Figure I Schematic diagram of the study. The self-assembled FA/Nano-Cur micelles composed of MPEG-PCL polymers, FA/MPEG-PCL polymers and Free Cur were administrated intravenously in the mice heterotopic colorectal cancer model, showing the capability of promoting tumor apoptosis, inhibiting tumor proliferation and attenuating tumor angiogenesis.

a nearly spherical morphology, indicating successful selfassembly of the polymers (Figure 2C). Furthermore, the DL and EE of FA/Cur-Nano were determined to be $10 \%$ and $98 \%$, respectively, by HPLC.

The drug release study is necessary for the evaluation of a drug delivery system. Thereupon, the release behavior of Cur from Free Cur, Nano-Cur and FA/NanoCur were investigated in our study. According to the in vitro drug release study, $91.60 \%, 44.53 \%, 46.32 \%$ of Cur has been released at $100 \mathrm{hrs}$ in Free Cur group, Nano-Cur group and FA/Nano-Cur group, respectively (Figure 2D). Compared with Free Cur group, a significantly slower release of Cur was observed in the Nano-Cur group and FA/Nano-Cur group, suggesting a more sustainable drug release. Moreover, the in vivo drug release study results showed elongated $T_{1 / 2}$ and increased $\mathrm{C}_{\max }$ as well as enlarged area under curve (AUC) by Nano-Cur and FA/Nano-Cur micelles. The $\mathrm{T}_{\max }, \mathrm{C}_{\max }, \mathrm{T}_{1 / 2}$ and AUC of Free Cur were $0.25 \mathrm{hrs}$, $61.67 \mu \mathrm{g} / \mathrm{mL}, 0.81 \mathrm{hrs}$ and $62.28 \mu \mathrm{g} \mathrm{mL}^{-1} \mathrm{hr}$. The $\mathrm{T}_{\max }$, $\mathrm{C}_{\max }, \mathrm{T}_{1 / 2}$ and AUC of Nano-Cur were $0.25 \mathrm{hrs}, 146.67$ $\mu \mathrm{g} / \mathrm{mL}, 1.42 \mathrm{hrs}$ and $478.6 \mu \mathrm{g} \mathrm{mL}^{-1} \mathrm{hr}$. The $\mathrm{T}_{\max }, \mathrm{C}_{\max }$, $\mathrm{T}_{1 / 2}$ and AUC of FA/Nano-Cur were $0.25 \mathrm{hrs}, 163.00$ $\mu \mathrm{g} / \mathrm{mL}, 1.58 \mathrm{hrs}$ and $579.1 \mu \mathrm{g} \mathrm{mL}^{-1} \mathrm{hr}$ (Figure 2E). The results showed that the nanoformulation of Cur significantly extended the drug duration of Cur agent.

\section{Cytotoxicity Assay}

We then investigated the cytotoxic effect of Free Cur, Nano-Cur and FA/Nano-Cur on CT26 cells using MTT assay. The results demonstrated that the cytotoxicity of Free Cur, Nano-Cur and FA/Nano-Cur were in dosedependent and time-dependent manners. At $24 \mathrm{hrs}$, the half-maximal inhibitory concentration $\left(\mathrm{IC}_{50}\right)$ of the Free Cur, Nano-Cur and FA/Nano-Cur were $9.614 \mu \mathrm{g} / \mathrm{mL}$, $5.683 \mu \mathrm{g} / \mathrm{mL}, 5.777 \mu \mathrm{g} / \mathrm{mL}$, respectively (Figure 3A). At $48 \mathrm{hrs}$, the $\mathrm{IC}_{50}$ of the Free Cur, Cur-Nano, FA/Cur-Nano was $9.490 \mu \mathrm{g} / \mathrm{mL}, 2.642 \mu \mathrm{g} / \mathrm{mL}, 1.373 \mu \mathrm{g} / \mathrm{mL}$, respectively (Figure 3B), which illustrated that FA/Nano-Cur exhibited the strongest cytotoxic effect compared with Free Cur and Nano-Cur in vitro study. 
A

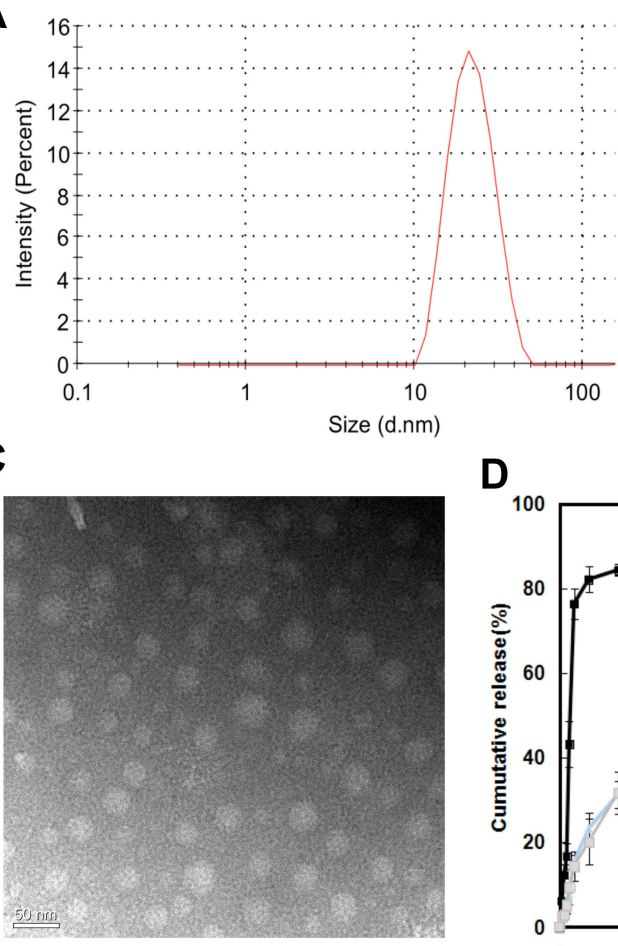

A

D

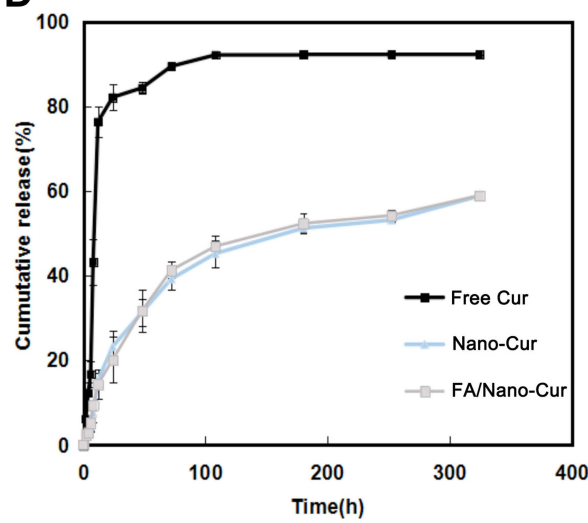

B

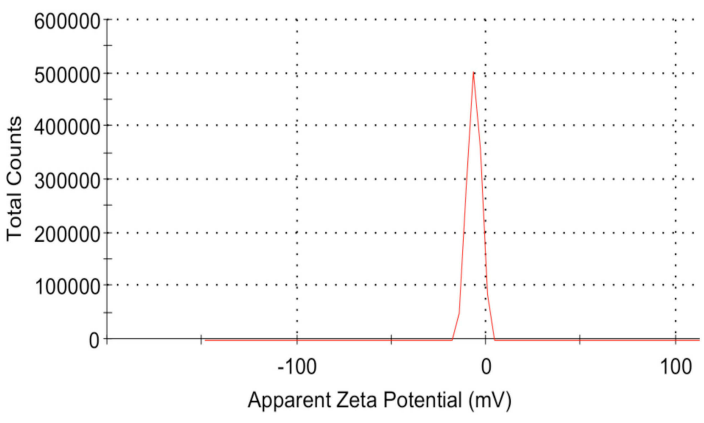

E

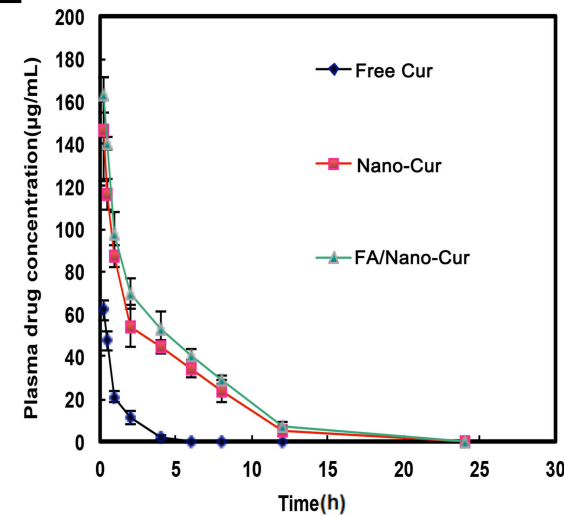

Figure 2 Characterization of FA/Nano-Cur micelles and drug release study. The particle size and the zeta-potential of FA/Nano-Cur micelles measured by the dynamic light scattering (DLS) method (A and B). Morphological identification of FA/Nano-Cur micelles under transmission electron microscopy (TEM) (C). Cur release from Free Cur, NanoCur micelles and FA/Nano-Cur micelles (D). Plasma drug concentration of Cur in Free Cur group, Nano-Cur group and FA/Nano-Cur group at different time point (E).
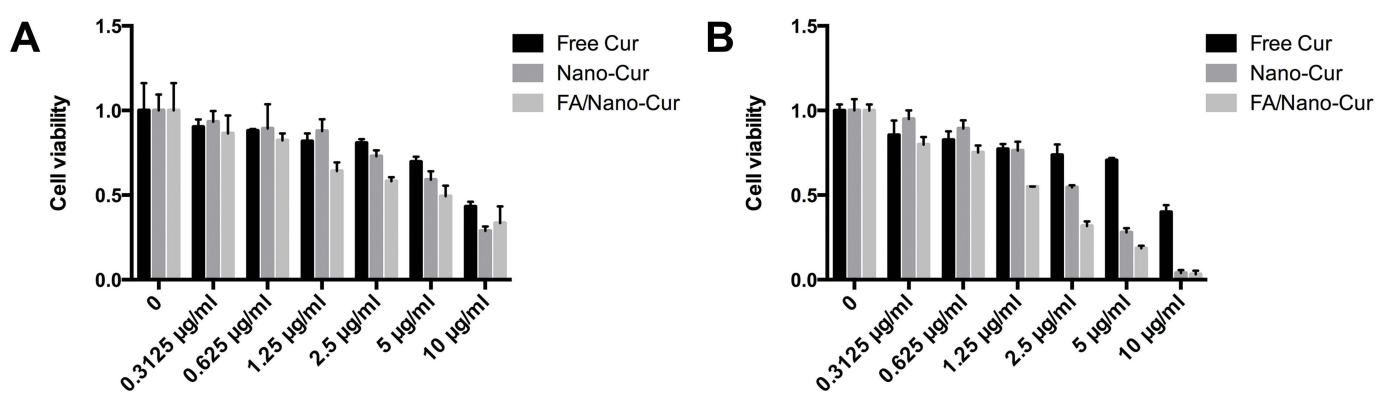

Figure 3 Cell cytotoxicity analysis. CT26 cells viability detected by MTT assay after treatment with different concentrations of Free Cur, Nano-Cur and FA/Nano-Cur at 24 hrs (A) and $48 \mathrm{hrs}$ (B)

\section{Cell Necrosis and Apoptosis Assay}

Many studies have already reported the pro-apoptotic effects of Cur on tumor cells. We performed PI/Annexin-V staining flow cytometry to determine the effects of Free Cur, Nano-Cur and FA/Nano-Cur on CT26 cells. The results indicated that CT26 cell death caused by Free Cur and Curloaded nanomicelles were mainly apoptosis. The percentage of Annexin- $\mathrm{V}^{+}$cells was $0.89 \%, 1.3 \%, 2.65 \%, 8.82 \%$ and $95.27 \%$ in the Free Cur treated group (Figure 4A), 1.22\%, $23.3 \%, 58.93 \%, 91.43 \%$ and $99.96 \%$ in the Nano-Cur treated group (Figure 4B), versus $0.87 \%, 51.53 \%, 50.18 \%, 96.59 \%$ and $99.95 \%$ in the FA/Nano-Cur treated group (Figure 4C) for concentrations of Cur at $0,3.125 \mu \mathrm{g} / \mathrm{mL}, 6.25 \mu \mathrm{g} / \mathrm{mL}$, $12.5 \mu \mathrm{g} / \mathrm{mL}$ and $25 \mu \mathrm{g} / \mathrm{mL}$, respectively. Hence, the above results confirmed that FA/Nano-Cur and Nano-Cur induced more cell apoptosis than Free Cur at the same concentration. Besides, there was an upper-shift of cell population with the increase of drug concentration, which might be attributed to the auto-fluorescence of Cur. It is noteworthy that the FA modification further enhanced the cytotoxicity of Nano-Cur, 

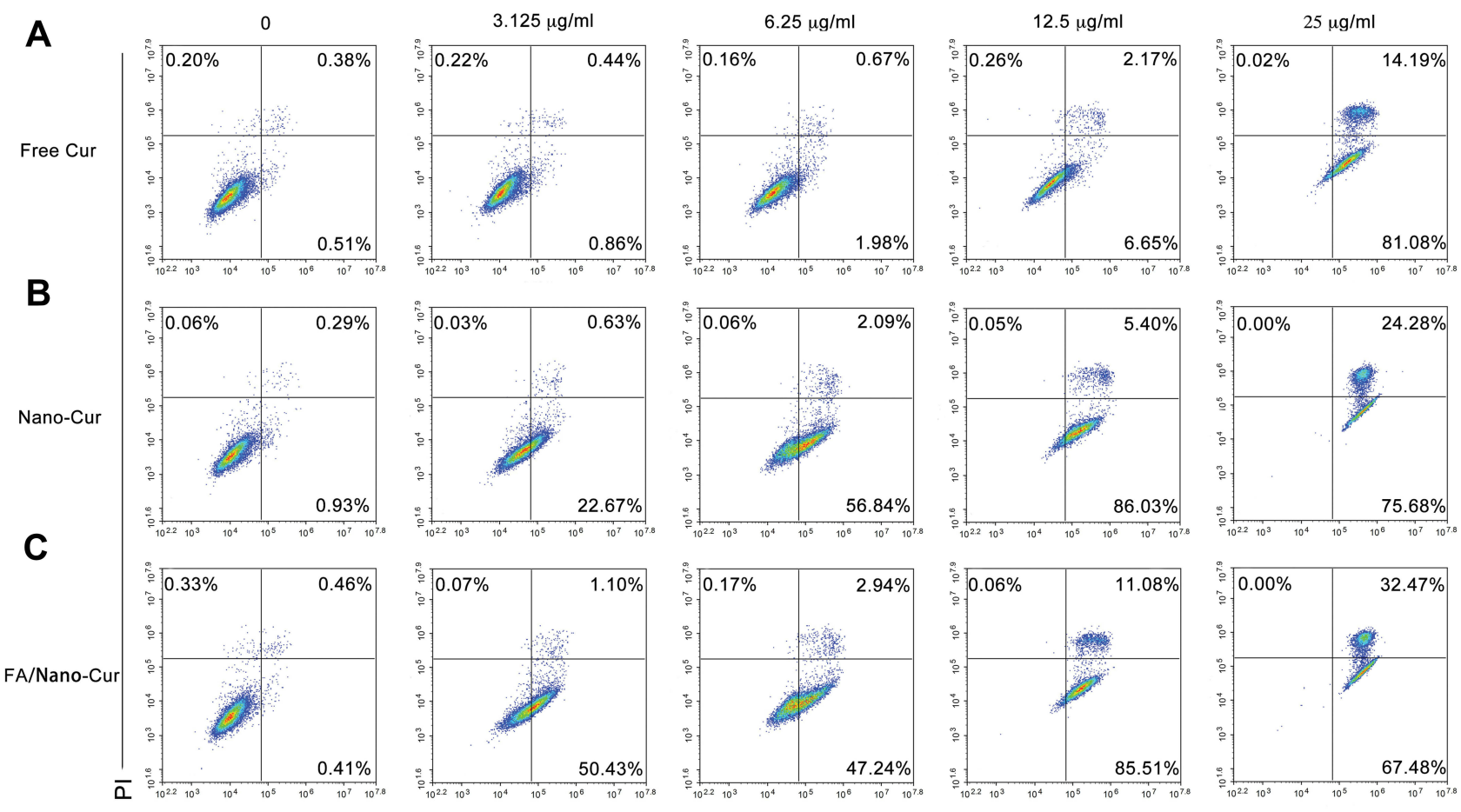

Annexin-V

Figure 4 Cell apoptosis and necrosis analysis. Flow cytometry results of PI/Annexin-V staining of CT26 cells treated with different concentrations of Free Cur (A), NanoCur (B) and FA/Nano-Cur (C) for 48 hrs.

demonstrated by more apoptotic cells in the FA/Nano-Cur treated group than that in the Nano-Cur treated group.

\section{In vivo Anti-Tumor Activity Therapeutic Efficacy in Mice Model of Heterotopic CRC}

The in vitro study results had found that FA/Nano-Cur and Nano-Cur showed enhanced ability to inhibit tumor proliferation and promote tumor apoptosis. Thereafter, a mice model of heterotopic CRC was established to evaluate the anti-tumor efficacy of different treatments in vivo. Figure 5A shows the subcutaneous tumors in each treatment group on the 18th day after tumor inoculation. The body weight of mice in each group increased gradually (Figure 5B). The tumor growth curves were plotted by dynamic monitoring of subcutaneous tumor volume, showing that the tumors in the control, FA/ Nano, Free Cur, and Nano-Cur groups grew rapidly, compared with slowly increase in the FA/Nano-Cur group from Day 12 to Day 18 (Figure 5C). On the 18th day, the tumor-inhibition rate was $77.32 \%$ in the FA/Cur-Nano group, $20.77 \%$ in the Nano-Cur group and $16.25 \%$ in the Free Cur group. No significant difference was shown between the FA/Nano group and control group. In addition, the comparison of tumor weight among different treatment groups validated the above conclusion (Figure 5D). Thus, our results demonstrated that Cur-loaded polymeric micelles were more effective than Free Cur, and in which FA/Nano-Cur exerted the strongest anti-tumor effect in the treatment of CRC.

\section{Effect of Inhibiting Tumor Proliferation}

In an anti-tumor drug assessment, it is essential to evaluate the ability of inhibiting tumor proliferation. In the study, the PCNA immunohistochemical staining was performed to compare the effect of different treatments on tumor proliferation. Our results showed that the PCNA index of Free Cur, Nano-Cur and FA/Nano-Cur were significantly lower than that of the control group and FA/Nano group, suggesting decreased tumor proliferation by Cur treatment (Figure 6A-E). Among all groups, the FA/Nano-Cur group had the fewest cells in proliferation status. Therefore, the results indicated that FA/Nano-Cur had a better effect on inhibiting tumor proliferation than other treatment groups.

\section{Effect of Inducing Tumor Cells Apoptosis}

Flow cytometry with PI/Annexin-V staining found that Free Cur, Nano-Cur and FA/Nano-Cur could induce tumor apoptosis in vitro. Hence, the TUNEL staining method was employed 
A

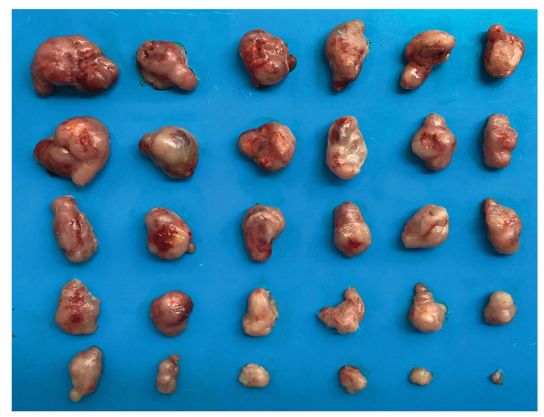

Control

FA/Nano

Free Cur

Nano-Cur

FA/Nano-Cur

C

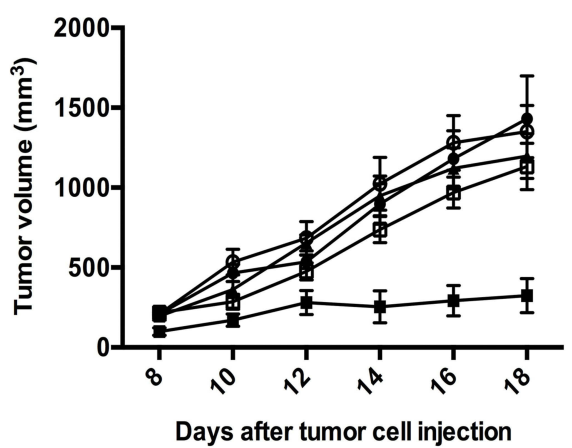

- FA/Nano-Cur

七 Nano-Cur

- Free Cur

- FA/Nano

$\rightarrow$ - Control
B

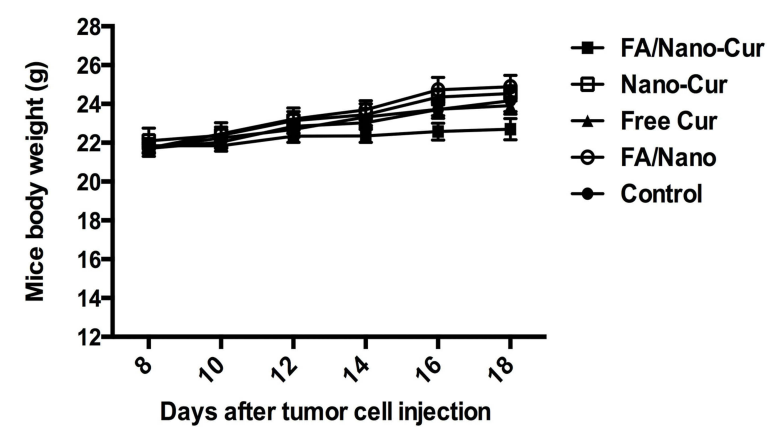

D

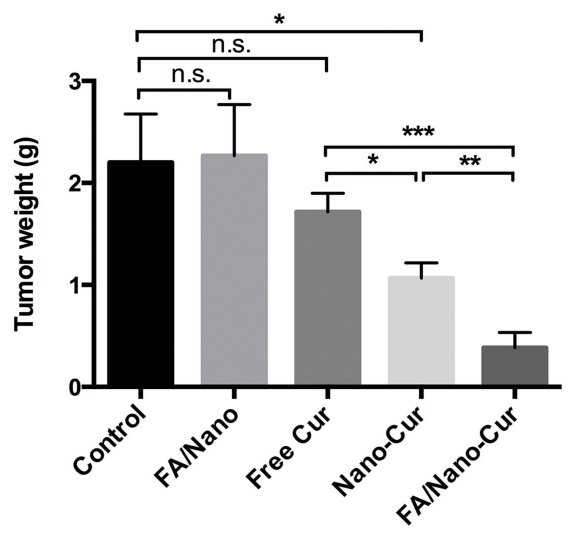

Figure $\mathbf{5}$ In vivo anti-tumor ability assessment of different treatments. The photograph of subcutaneous tumors from different treatment groups (A), the boy weight change curve of mice in different groups (B), the tumor growth curve of mice from different treatment groups (C) and the tumor weight of mice from different treatment groups (D) $(* \mathrm{P}<0.05, * * \mathrm{P}<0.01, * * * \mathrm{P}<0.00 \mathrm{I})$.

A

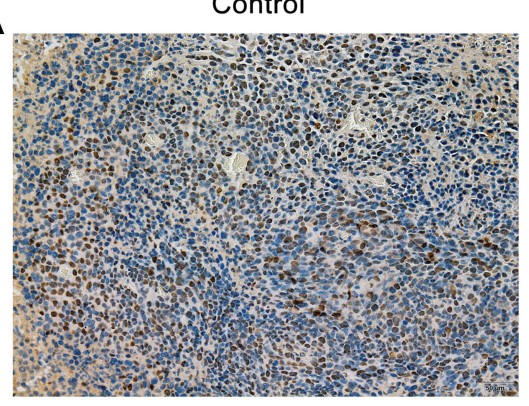

D

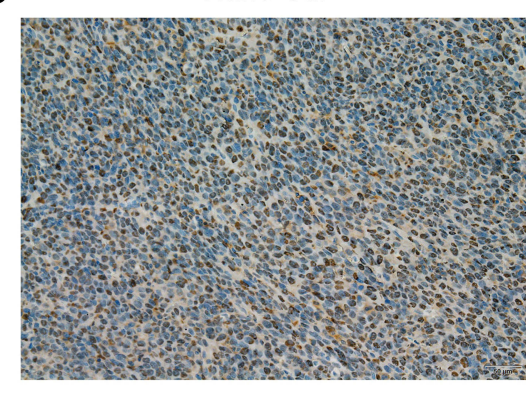

B

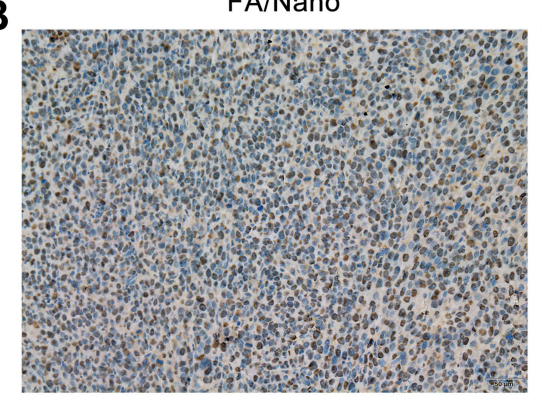

E

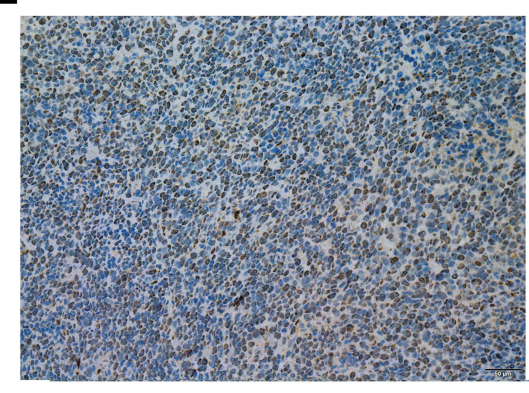

Free Cur
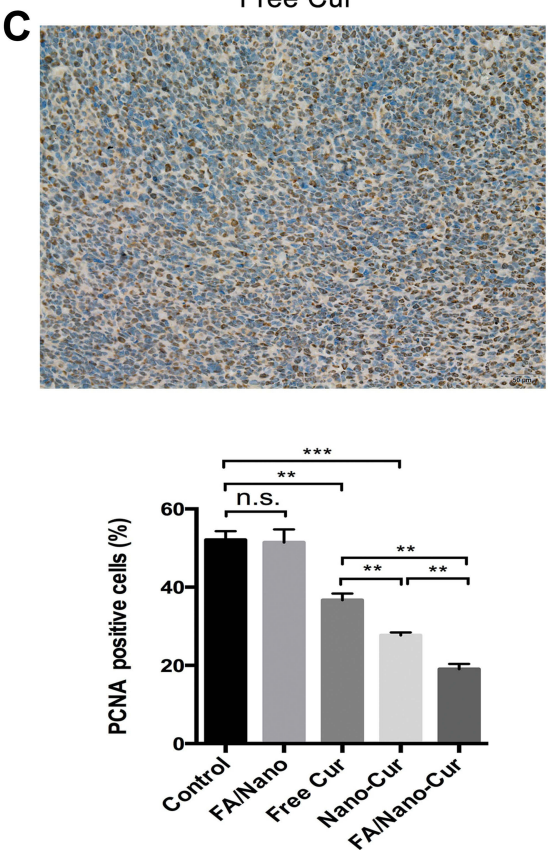

Figure 6 Effect on tumor proliferation. PCNA staining positive cells percentage in Control group (A), FA/Nano group (B), Free Cur group (C), Nano-Cur group (D) and FA/Nano-Cur group $(\mathrm{E})(* * \mathrm{P}<0.01, * * * \mathrm{P}<0.001)$. 
to study the effect of different treatments on tumor apoptosis in vivo. As a result, only few apoptotic cells were observed in the control group and the FA/Nano group whereas the apoptotic cells increased slightly in the Free Cur group (Figure 7A-E). There was a large number of apoptotic cells observed in the Nano-Cur treated group and the FA/Nano-Cur treated group, and the proportion of apoptotic cells in the FA/ Nano-Cur group was higher than that of the Nano-Cur group. Taken together, the results confirmed that FA/Nano-Cur could induce more tumor cells apoptosis, thereby enhancing the antitumor effect.

\section{Effect of Attenuating Tumor Angiogenesis}

Tumor angiogenesis is crucial for the development and metastasis of malignant tumor. To further explore the antiangiogenesis capability of different treatments, we labeled the vascular endothelial cells in tumor tissues using immunohistochemical staining method. The number of CD31 positive cells in the randomly selected microscopic field was defined as microvessel density (MVD). Results showed that the MVD in all but the FA/Nano group was significantly lower than the control group, and the FA/Nano-Cur group had the minimum MVD (Figure 8A-E). In addition to inhibiting proliferation and promoting apoptosis, it was demonstrated that Free Cur,
Nano-Cur and FA/Nano-Cur exhibited the capability of antiangiogenesis, among which FA/Nano-Cur was the most effective one.

\section{Systemic Toxicity Assessment}

In order to investigate the systemic toxicity of different treatments, the vital organs including heart, liver, spleen, lung and kidney of mice from each treatment group were sliced and examined by $\mathrm{HE}$ staining. No apparent morphological abnormalities were found in all treatment groups (Figure 9A-E). Furthermore, the appetite change, diarrhea and abnormal behaviors of mice were monitored to evaluate the adverse effects. No obvious appetite and behavioral abnormalities appeared in the groups throughout the treatment period. Besides, there was no significant difference in serological biochemical parameters among the groups, suggesting that all treatments were low toxic to the mice (Figure 10A-D). The FA/Nano-Cur and Nano-Cur prepared in the study showed good safety, which indicated their great potential for future application.

\section{Discussion}

As the natural product extracted from traditional Asian spice Curcuma longa L., Cur is easily available, low toxic, and
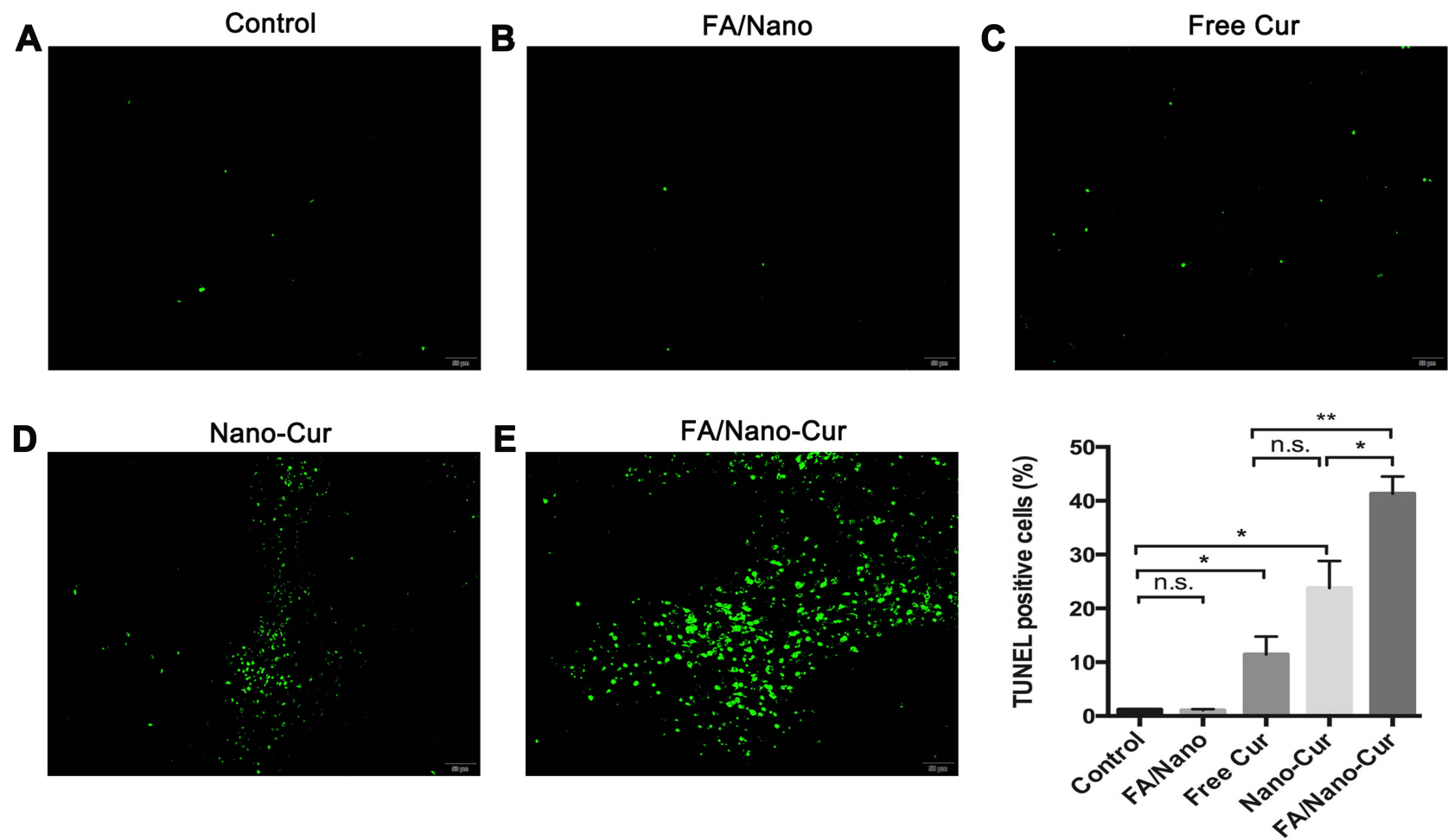

Figure 7 Effect on tumor apoptosis. The results of TUNEL staining in Control group (A), FA/Nano-Cur group (B), Free Cur group (C), Nano-Cur group (D) and FA/NanoCur group (E) $(* \mathrm{P}<0.05, * * \mathrm{P}<0.0 \mathrm{I})$. 

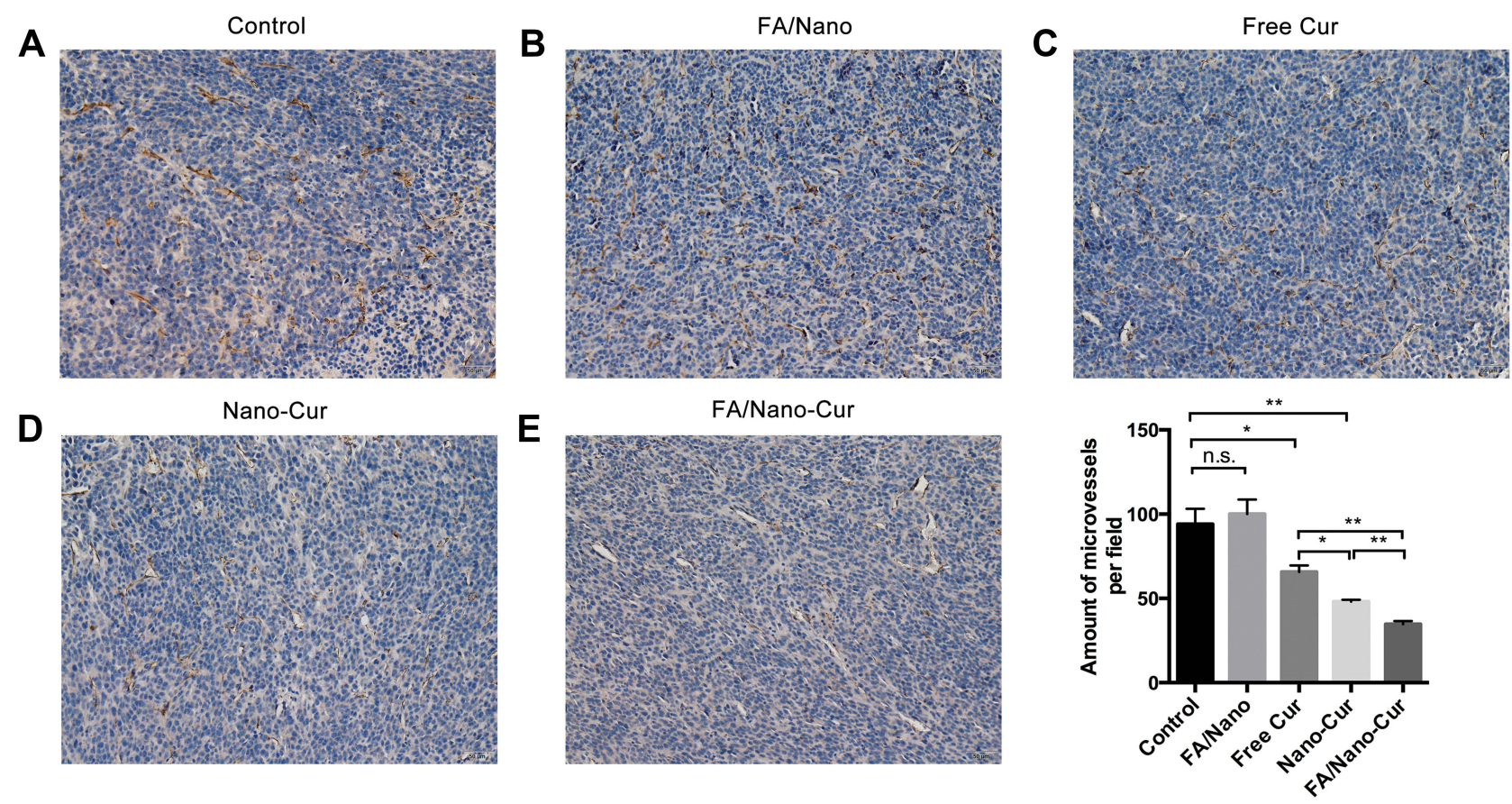

Figure 8 Effect on tumor vascularization. Microvessel densities in Control group (A), FA/Nano group (B), Free Cur group (C), Nano-Cur group (D) and FA/Nano-Cur group $(\mathbf{E})(* \mathrm{P}<0.05, * * \mathrm{P}<0.01)$.

mass-produced. Cur has received extensive attention for its versatile role in many pharmacological activities, which makes it a promising drug candidate in the treatment of various diseases. ${ }^{13,14,16-18}$ The anti-tumor mechanisms of Cur were associated with the development and progression of cancer. Many signal pathways were involved in the processes including mitogen-activated protein kinase (MAPK), peroxisome proliferator-activated receptor (PPAR), WNT/ beta-catenin, sonic hedgehog (sHh), NOTCH and PI3K/ PTEN/Akt/mTORC, protein 53 (P53), activating protein 1 (AP-1), signal transducers and activators of transcription 3 (STAT3), B-cell lymphoma-2 (Bcl-2), epidermal growth response-1 (Egr-1), showing the multi-target capability of Cur in cancer treatment. ${ }^{41-47}$ In CRC, Cur was also able to suppress cyclooxygenase-2 (COX-2) and reduce prostaglandin synthesis in the colon carcinogenesis process. ${ }^{48,49}$

One previous study has demonstrated that consuming 8 $\mathrm{g}$ of Cur per day for 3 months caused no significant toxic effect in patients with high-risk or precancerous lesions. ${ }^{50}$ However, the solubility of Cur was reported to be less than $0.125 \mathrm{mg} / \mathrm{L}$ in water. ${ }^{51}$ What is more, the content of Cur was undetectable in mice plasma and brain at $0.5 \mathrm{~h}$ after oral administration of Cur (50 mg/kg), indicating poor bioavailability. ${ }^{52}$ Research has shown that the content of Cur was merely $50 \mathrm{ng} / \mathrm{mL}$ after oral administration of Cur at the dosage of $10-12 \mathrm{mg} / \mathrm{kg}$ in human. ${ }^{53}$ Some researches reported FA modified MPEG-PCL polymers encapsulating chemotherapeutic or hormonetherapeutic drugs such as doxorubicin, paclitaxel and tamoxifen in cancer therapy, which showed a good anti-tumor effect. ${ }^{54-56}$ Another study introduced a Y-shaped FA-MPEG$\mathrm{PCL}_{2}$ copolymer with a particle size of $48.94 \mathrm{~nm}$ to improve the uptake of Cur in Hela and HepG2 cells. ${ }^{57}$ Herein, we synthesized Cur-loading FA/MPEG-PCL micelles (FA/NanoCur) with an average size of $30.47 \mathrm{~nm}$ for vein injection, which was expected to improve the bioavailability of Cur. Gou et al applied MPEG-PCL micelles in the Cur drug delivery system for CRC treatment, which showed an improved anti-tumor effect than free Cur in mice model. ${ }^{34}$ In the research, we used FA conjugated MPEG-PCL micelle to encapsulate hydrophobic Cur. The drug release study showed the nanoformulation of Cur significantly extended drug duration of Cur and had a prominent delayed drug release effect. Improved $T_{1 / 2}$ and increased $C_{\max }$ as well as the enlarged area under curve (AUC) by Nano-Cur and FA/Nano-Cur micelles were observed. FA/Nano-Cur not only possessed the outstanding properties of nanoscale MPEG-PCL micelles, such as improving water solubility, sustaining release, reducing systemic elimination, enhanced permeability and retention (EPR) effect, but also exhibited enhanced tumor-targeting capability, which further augmented the therapeutic efficacy. Taken together, the low toxicity of Cur and the selective delivery by FA conjugated nanoformulation would reduce adverse 


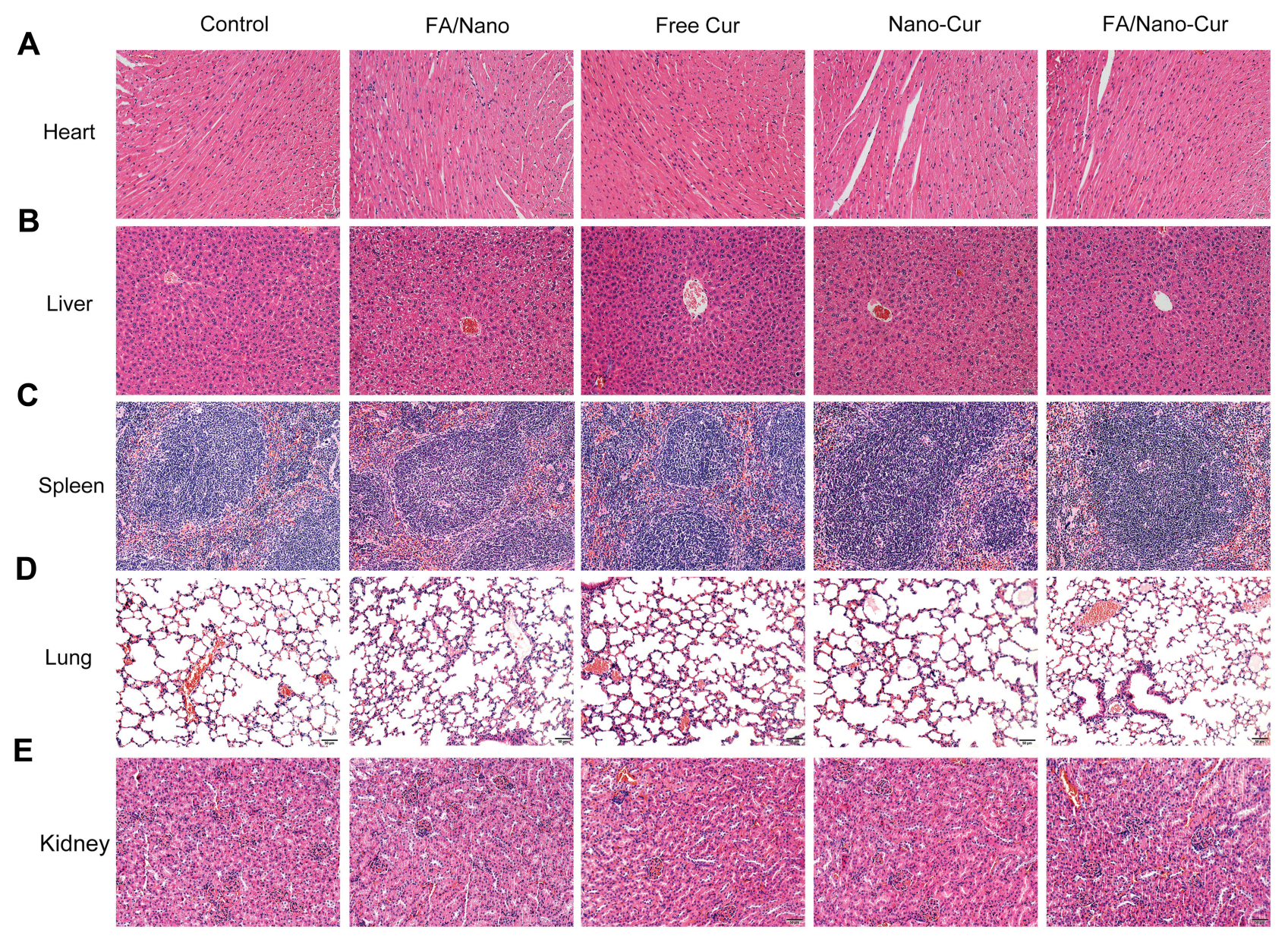

Figure 9 Safety assessment on vital organs. HE staining results of heart (A), Liver (B), spleen (C), lung (D) and kidney (E) sections from different treatment groups were presented.

effects, compared with the systemic diffusion of chemotherapeutic drug.

Our results demonstrated Free Cur, Nano-Cur and FA/ Nano-Cur were capable of inducing colon tumor cell apoptosis both in vitro and in vivo, and FA/Nano-Cur exhibited the most powerful effect. Cur has been disclosed to induce cell apoptosis via suppressing NF- $\mathrm{kB}$ and its associated pathways. ${ }^{44}$ Cur could also promote apoptosis through activating cancer suppressor gene p53 and down-regulating anti-apoptotic genes Bcl- 2/Bcl-xL as well as upregulating pro-apoptotic genes Bax. ${ }^{47}$ Furthermore, Cur was found to induce growth arrest and apoptosis by affecting Wnt and cell-cell adhesion pathway. ${ }^{43}$ In addition, we found the antiproliferation effect of Free Cur and Cur nanoformulation by MTT assay and PCNA staining method. Research has reported Cur might suppress the expression of epidermal growth factor receptor (EGFR) by reducing Egr-1 activity in colon cancer cells, thus to restrain colon cancer cell growth. ${ }^{41}$ The tumor angiogenesis in FA/Nano-Cur treated group was significantly mitigated as well. Cur was reported to potentiate the anti-tumor effect of gemcitabine in an orthotopic model of pancreatic cancer by reducing $\mathrm{CD} 31^{+}$ microvessel density. ${ }^{45}$ In summary, the results illustrated that FA/Nano-Cur treatment exhibited stronger anti-tumor ability in terms of promoting tumor cell apoptosis, inhibiting tumor cell growth and reducing tumor angiogenesis, compared with Nano-Cur treatment. Besides, no obvious systemic toxicity induced by FA/Nano-Cur injection was found through serological detection and pathomorphological observation.

So far, there are three ongoing clinical trials on Cur nanoformulation in the treatment of CRC and colorectal adenomatous polyps, indicating promising prospects of $\mathrm{FA} /$ Nano-Cur application in future. ${ }^{58}$ Still, great efforts are required to ascertain the therapeutic effect and toxicity of FA/Nano-Cur for human CRC therapy. Research has reported that Cur showed a synergistic effect with 5-fluorouracil in gastric cancer. ${ }^{59}$ Cur could also sensitize human 


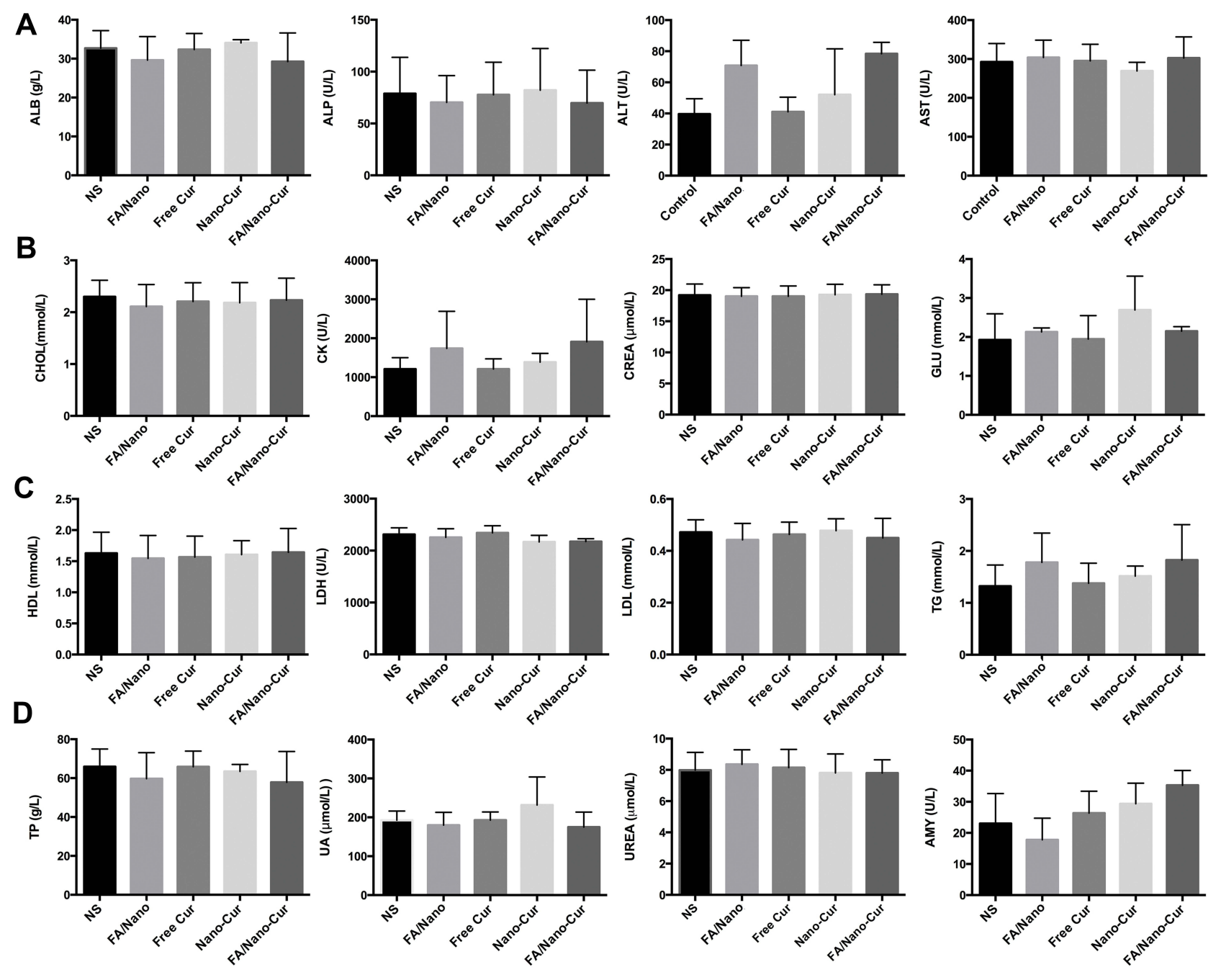

Figure 10 Serological biochemical parameters detection. A series of biochemical parameters in mice serum from different groups were detected including ALB, ALP, ALT, AST (A), CHOL, CK, CREA, GLU (B), HDL, LDH, LDL, TG (C) and TP, UA, UREA, AMY (D).

CRC xenograft to gamma-radiation. ${ }^{60}$ We hope our study will provide a new aspect for CRC treatment and more research are warranted to investigate the therapeutic effect of FA/Nano-Cur on other malignant cancer types. The combination therapy of FA/Nano-Cur and other treatment is also an interesting and meaningful topic for future study.

\section{Conclusion}

We prepared a new injectable dosage form of Cur formulation by encapsulating Cur in FA modified MPEG-PCL micelles. Our preclinical study in the CRC mice model has shown that FA/Nano-Cur exhibited a significantly better therapeutic effect than Free Cur and Nano-Cur. FA/Nano-Cur treatment induced tumor cell apoptosis while significantly reduced the tumor proliferation and tumor angiogenesis, which might be attributed to improved bioavailability and enhanced tumor- targeting effect. Taken together, FA/Nano-Cur is a promising therapeutic agent for CRC treatment with low toxicity and better efficacy as well as good biodegradability.

\section{Acknowledgment}

This research was supported by the General Research Program of the Science and Technology Department of Sichuan Province (2018FZ0094) and the National Natural Science Foundation of China (81972347).

\section{Disclosure}

The authors report no conflicts of interest in this work.

\section{References}

1. Brenner H, Kloor M, Pox CP. Colorectal cancer. Lancet. 2014;383 (9927):1490-1502. doi:10.1016/S0140-6736(13)61649-9 
2. Center MM, Jemal A, Smith RA, Ward E. Worldwide variations in colorectal cancer. CA Cancer J Clin. 2009;59(6):366-378. doi:10.3322/caac. 20038

3. Center MM, Jemal A, Ward E. International trends in colorectal cancer incidence rates. Cancer Epidemiol Biomarkers Prev. 2009;18 (6):1688-1694. doi:10.1158/1055-9965.EPI-09-0090

4. Siegel RL, Miller KD, Fedewa SA, et al. Colorectal cancer statistics, 2017. CA Cancer J Clin. 2017;67(3):177-193. doi:10.3322/caac.21395

5. Chan DS, Aune D, Vieira R, Greenwood DC, Kampman E, Norat T. Red and processed meat and colorectal cancer incidence: meta-analysis of prospective studies. PLoS One. 2011;6(6):e20456. doi:10.1371/journal.pone.0020456

6. Edwards BK, Ward E, Kohler BA, et al. Annual report to the nation on the status of cancer, 1975-2006, featuring colorectal cancer trends and impact of interventions (risk factors, screening, and treatment) to reduce future rates. Cancer. 2010;116(3):544-573. doi:10.1002/cncr.v116:3

7. Fedirko V, Tramacere I, Bagnardi V, et al. Alcohol drinking and colorectal cancer risk: an overall and dose-response meta-analysis of published studies. Ann Oncol. 2011;22(9):1958-1972. doi:10.1093/annonc/ mdq653

8. Liang PS, Chen TY, Giovannucci E. Cigarette smoking and colorectal cancer incidence and mortality: systematic review and meta-analysis. Int $J$ Cancer. 2009;124(10):2406-2415. doi:10.1002/ijc.24191

9. Ma Y, Yang Y, Wang F, et al. Obesity and risk of colorectal cancer: a systematic review of prospective studies. PLoS One. 2013;8(1): e53916. doi:10.1371/journal.pone.0053916

10. Siegel R, DeSantis C, Virgo K, et al. Cancer treatment and survivorship statistics, 2012. CA Cancer J Clin. 2012;62(4):220-241. doi:10.3322/caac.v62:4

11. Di Maio M, Basch E, Bryce J, Perrone F. Patient-reported outcomes in the evaluation of toxicity of anticancer treatments. Nat Rev Clin Oncol. 2016;13(5):319-325. doi:10.1038/nrclinonc.2015.222

12. Ajazuddin, Alexander A, Qureshi A. Role of herbal bioactives as a potential bioavailability enhancer for active pharmaceutical ingredients. Fitoterapia. 2014;97:1-14. doi:10.1016/j.fitote.2014.05.005

13. Aggarwal BB. Targeting inflammation-induced obesity and metabolic diseases by curcumin and other nutraceuticals. Annu Rev Nutr. 2010;30:173-199. doi:10.1146/annurev.nutr.012809.104755

14. Allegra A, Innao V, Russo S, Gerace D, Alonci A, Musolino C. Anticancer activity of curcumin and its analogues: preclinical and clinical studies. Cancer Invest. 2017;35(1):1-22. doi:10.1080/ 07357907.2016.1247166

15. Anand P, Sundaram C, Jhurani S, Kunnumakkara AB, Aggarwal BB. Curcumin and cancer: an "old-age" disease with an "age-old" solution. Cancer Lett. 2008;267(1):133-164. doi:10.1016/j.canlet.2008. 03.025

16. Jagetia GC, Aggarwal BB. "Spicing up" of the immune system by curcumin. J Clin Immunol. 2007;27(1):19-35. doi:10.1007/s10875006-9066-7

17. Maheshwari RK, Singh AK, Gaddipati J, Srimal RC. Multiple biological activities of curcumin: a short review. Life Sci. 2006;78 (18):2081-2087. doi:10.1016/j.1fs.2005.12.007

18. Shin SK, Ha TY, McGregor RA, Choi MS. Long-term curcumin administration protects against atherosclerosis via hepatic regulation of lipoprotein cholesterol metabolism. Mol Nutr Food Res. 2011;55 (12):1829-1840. doi:10.1002/mnfr.201100440

19. Bar-Sela G, Epelbaum R, Schaffer M. Curcumin as an anti-cancer agent: review of the gap between basic and clinical applications. Curr Med Chem. 2010;17(3):190-197. doi:10.2174/092986710790149738

20. Duvoix A, Blasius R, Delhalle S, et al. Chemopreventive and therapeutic effects of curcumin. Cancer Lett. 2005;223(2):181-190. doi:10.1016/j.canlet.2004.09.041

21. Ide H, Tokiwa S, Sakamaki K, et al. Combined inhibitory effects of soy isoflavones and curcumin on the production of prostate-specific antigen. Prostate. 2010;70(10):1127-1133. doi:10.1002/pros.v70:10
22. Kakarala M, Brenner DE, Korkaya H. Targeting breast stem cells with the cancer preventive compounds curcumin and piperine. Breast Cancer Res Treat. 2010;122(3):777-785. doi:10.1007/s10549-0090612-x

23. Ravindran J, Prasad S, Aggarwal BB. Curcumin and cancer cells: how many ways can curry kill tumor cells selectively? AAPS J. 2009;11(3):495-510. doi:10.1208/s12248-009-9128-x

24. Soleimani V, Sahebkar A, Hosseinzadeh H. Turmeric (Curcuma longa) and its major constituent (curcumin) as nontoxic and safe substances: review. Phytother Res. 2018;32(6):985-995. doi:10.100 2/ptr.v32.6

25. Hewlings SJ, Kalman DS. Curcumin: a review of its' effects on human health. Foods. 2017;6(10):E92. doi:10.3390/foods6100092

26. Liu W, Zhai Y, Heng X, et al. Oral bioavailability of curcumin: problems and advancements. J Drug Target. 2016;24(8):694-702. doi:10.3109/1061186X.2016.1157883

27. Yang KY, Lin LC, Tseng TY, Wang SC, Tsai TH. Oral bioavailability of curcumin in rat and the herbal analysis from Curcuma longa by LC-MS/MS. J Chromatogr B Analyt Technol Biomed Life Sci. 2007;853(1-2):183-189. doi:10.1016/j.jchromb.2007.03.010

28. Hu Y, Wu C, Zhu C, et al. Enhanced uptake and improved anti-tumor efficacy of doxorubicin loaded fibrin gel with liposomal apatinib in colorectal cancer. Int J Pharm. 2018;552(1-2):319-327. doi:10.1016/ j.ijpharm.2018.10.013

29. Sinha R, Kim G, Nie S, Shin D. Nanotechnology in cancer therapeutics: bioconjugated nanoparticles for drug delivery. Mol Cancer Ther. 2006;5(8):1909-1917. doi:10.1158/1535-7163.MCT-06-0141

30. Liu X, Li Y, Sun X, et al. Powerful anti-colon cancer effect of modified nanoparticle-mediated IL-15 immunogene therapy through activation of the host immune system. Theranostics. 2018;8 (13):3490-3503. doi:10.7150/thno.24157

31. Danafar H, Sharafi A, Kheiri Manjili H, Andalib S. Sulforaphane delivery using mPEG-PCL co-polymer nanoparticles to breast cancer cells. Pharm Dev Technol. 2017;22(5):642-651. doi:10.3109/ 10837450.2016.1146296

32. Zamani M, Shirinzadeh A, Aghajanzadeh M, Andalib S, Danafar H. In vivo study of mPEG-PCL as a nanocarriers for anti-inflammatory drug delivery of simvastatin. Pharm Dev Technol. 2019;24 (6):663-670. doi:10.1080/10837450.2018.1556689

33. Gou M, Men K, Shi H, et al. Curcumin-loaded biodegradable polymeric micelles for colon cancer therapy in vitro and in vivo. Nanoscale. 2011;3(4):1558-1567. doi:10.1039/c0nr00758g

34. Gou M, Wei X, Men K, et al. PCL/PEG copolymeric nanoparticles: potential nanoplatforms for anticancer agent delivery. Curr Drug Targets. 2011;12(8):1131-1150. doi:10.2174/138945011795906642

35. Lammers T, Kiessling F, Hennink WE, Storm G. Drug targeting to tumors: principles, pitfalls and (pre-) clinical progress. J Control Release. 2012;161(2):175-187. doi:10.1016/j.jconrel.2011.09.063

36. Leamon CP, Reddy JA. Folate-targeted chemotherapy. Adv Drug Deliv Rev. 2004;56(8):1127-1141. doi:10.1016/j.addr.2004.01.008

37. Antony AC. Folate receptors: reflections on a personal odyssey and a perspective on unfolding truth. Adv Drug Deliv Rev. 2004;56 (8):1059-1066. doi:10.1016/j.addr.2004.01.007

38. Elnakat H, Ratnam M. Distribution, functionality and gene regulation of folate receptor isoforms: implications in targeted therapy. $A d v$ Drug Deliv Rev. 2004;56(8):1067-1084. doi:10.1016/j.addr.2004. 01.001

39. Liu X, Wang B, Li Y, et al. Powerful anticolon tumor effect of targeted gene immunotherapy using folate-modified nanoparticle delivery of CCL19 to activate the immune system. ACS Cent Sci. 2019;5(2):277-289. doi:10.1021/acscentsci.8b00688

40. Parker N, Turk MJ, Westrick E, Lewis JD, Low PS, Leamon CP. Folate receptor expression in carcinomas and normal tissues determined by a quantitative radioligand binding assay. Anal Biochem. 2005;338(2):284-293. doi:10.1016/j.ab.2004.12.026 
41. Chen A, Xu J, Johnson AC. Curcumin inhibits human colon cancer cell growth by suppressing gene expression of epidermal growth factor receptor through reducing the activity of the transcription factor Egr-1. Oncogene. 2006;25(2):278-287. doi:10.1038/sj.onc.1209019

42. Ghosh AK, Kay NE, Secreto CR, Shanafelt TD. Curcumin inhibits prosurvival pathways in chronic lymphocytic leukemia B cells and may overcome their stromal protection in combination with EGCG. Clin Cancer Res. 2009;15(4):1250-1258. doi:10.1158/1078-0432. CCR-08-1511

43. Jaiswal AS, Marlow BP, Gupta N, Narayan S. Beta-catenin-mediated transactivation and cell-cell adhesion pathways are important in curcumin (diferuylmethane)-induced growth arrest and apoptosis in colon cancer cells. Oncogene. 2002;21(55):8414-8427. doi:10.1038/ sj.onc. 1205947

44. Kunnumakkara AB, Bordoloi D, Harsha C, Banik K, Gupta SC, Aggarwal BB. Curcumin mediates anticancer effects by modulating multiple cell signaling pathways. Clin Sci. 2017;131(15):1781-1799. doi:10.1042/CS20160935

45. Kunnumakkara AB, Guha S, Krishnan S, Diagaradjane P, Gelovani J, Aggarwal BB. Curcumin potentiates antitumor activity of gemcitabine in an orthotopic model of pancreatic cancer through suppression of proliferation, angiogenesis, and inhibition of nuclear factor-kappaBregulated gene products. Cancer Res. 2007;67(8):3853-3861. doi:10.1158/0008-5472.CAN-06-4257

46. Patel BB, Sengupta R, Qazi S, et al. Curcumin enhances the effects of 5-fluorouracil and oxaliplatin in mediating growth inhibition of colon cancer cells by modulating EGFR and IGF-1R. Int $J$ Cancer. 2008;122(2):267-273. doi:10.1002/ijc.23097

47. Sa G, Das T. Anti cancer effects of curcumin: cycle of life and death. Cell Div. 2008;3:14. doi:10.1186/1747-1028-3-14

48. Goel A, Boland C, Chauhan DP. Specific inhibition of cyclooxygenase-2 (COX-2) expression by dietary curcumin in HT-29 human colon cancer cells. Cancer Lett. 2001;172(2):111-118. doi:10.1016/S0304-3835(01) 00655-3

49. Zhang F, Altorki NK, Mestre JR, Subbaramaiah K, Dannenberg AJ. Curcumin inhibits cyclooxygenase- 2 transcription in bile acid- and phorbol ester-treated human gastrointestinal epithelial cells. Carcinogenesis. 1999;20(3):445-451. doi:10.1093/carcin/20.3.445

50. Cheng AL, Hsu CH, Lin JK, et al. Phase I clinical trial of curcumin, a chemopreventive agent, in patients with high-risk or pre-malignant lesions. Anticancer Res. 2001;21(4B):2895-2900.
51. Anand P, Kunnumakkara AB, Newman RA, Aggarwal BB. Bioavailability of curcumin: problems and promises. Mol Pharm. 2007;4(6):807-818. doi:10.1021/mp700113r

52. Schiborr C, Rimbach G, Frank J. A validated method for the quantification of curcumin in plasma and brain tissue by fast narrow-bore high-performance liquid chromatography with fluorescence detection. Anal Bioanal Chem. 2010;397(5):1917-1925. doi:10.1007/s00216-0103719-3

53. Kaminaga Y, Nagatsu A, Akiyama T, et al. Production of unnatural glucosides of curcumin with drastically enhanced water solubility by cell suspension cultures of Catharanthus roseus. FEBS Lett. 2003;555 (2):311-316. doi:10.1016/S0014-5793(03)01265-1

54. Zhang L, Zhu D, Dong X, et al. Folate-modified lipid-polymer hybrid nanoparticles for targeted paclitaxel delivery. Int $J$ Nanomedicine. 2015;10:2101-2114. doi:10.2147/IJN.S77667

55. Zamani M, Rostamizadeh K, Manjili H, Danafar $H$. In vitro and in vivo biocompatibility study of folate-lysine-PEG-PCL as nanocarrier for targeted breast cancer drug delivery. Eur Polym J. 2018;103:260-270. doi:10.1016/j.eurpolymj.2018.04.020

56. Cuong NV, Li YL, Hsieh MF. Targeted delivery of doxorubicin to human breast cancers by folate-decorated star-shaped PEG-PCL micelle. J Mater Chem. 2011;22(3):1006-1020. doi:10.1039/C1JM13588K

57. Feng R, Zhu W, Chu W, et al. Y-shaped folic acid-conjugated PEG-PCL copolymeric micelles for delivery of curcumin. Anticancer Agents Med Chem. 2017;17(4):599-607. doi:10.2174/ 1871520616666160815124014

58. Wong KE, Ngai SC, Chan KG, Lee LH, Goh BH, Chuah LH. Curcumin nanoformulations for colorectal cancer: a review. Front Pharmacol. 2019;10:152. doi:10.3389/fphar.2019.00152

59. Koo JY, Kim HJ, Jung KO, Park KY. Curcumin inhibits the growth of AGS human gastric carcinoma cells in vitro and shows synergism with 5-fluorouracil. J Meds Food. 2004;7(2):117-121. doi:10.1089/ 1096620041224229

60. Kunnumakkara AB, Diagaradjane P, Guha S, et al. Curcumin sensitizes human colorectal cancer xenografts in nude mice to gamma-radiation by targeting nuclear factor-kappaB-regulated gene products. Clin Cancer Res. 2008;14(7):2128-2136. doi:10.1158/ 1078-0432.CCR-07-4722
International Journal of Nanomedicine

\section{Publish your work in this journal}

The International Journal of Nanomedicine is an international, peerreviewed journal focusing on the application of nanotechnology in diagnostics, therapeutics, and drug delivery systems throughout the biomedical field. This journal is indexed on PubMed Central, MedLine, CAS, SciSearch ${ }^{\mathbb{R}}$, Current Contents ${ }^{\mathbb{R}} /$ Clinical Medicine, $^{2}$

\section{Dovepress}

Journal Citation Reports/Science Edition, EMBase, Scopus and the Elsevier Bibliographic databases. The manuscript management system is completely online and includes a very quick and fair peer-review system, which is all easy to use. Visit http://www.dovepress.com/ testimonials.php to read real quotes from published authors. 